\title{
AOR
}

Selected Papers of \#AolR2020:

The $21^{\text {st }}$ Annual Conference of the

Association of Internet Researchers

Virtual Event / 27-31 October 2020

\section{SOCIALITY AND MONETIZATION ON LIVE STREAMING PLATFORMS}

\author{
Mark R Johnson PhD \\ University of Sydney \\ Jamie Woodcock PhD \\ The Open University \\ Nick Bowman PhD \\ Texas Tech University \\ Will Partin \\ University of North Carolina at Chapel Hill \\ Nathan Jackson \\ University of New South Wales
}

\section{Introductory Statement}

This panel presents an overview of ongoing research into online "live streaming" platforms, which offer the live broadcast of individuals' activities - primarily but not exclusively digital gameplay - over the internet to potentially massive worldwide audiences. The largest platform in this area (on which we focus) is Twitch.tv, already the 30th most-viewed website in the world, with comparable platforms boasting large viewing numbers in China, Korea, and Japan. Marking live streaming out from other forms of online media is the potential for rapid back-and-forth communication between "streamers" and their potentially tens of thousands of concurrent viewers, with often only a few seconds of time lag. Equally, much of this communication is tied to impressive volume of altruistic financial support from loyal viewers, with the most successful of the world's several thousand full-time professional live streamers bringing in several million USD per year. Live streaming is a major growing interdisciplinary area of internet research, drawing primarily on media studies but also scholars from psychology,

Suggested Citation (APA): Johnson, M. R.; Woodcock, J.; Bowman, N.; Partin, W. \& Jackson, N. (2020, October 28-31). Sociality and Monetization on Live Streaming Platforms. Panel presented at AoIR 2020: The $21^{\text {th }}$ Annual Conference of the Association of Internet Researchers. Dublin, Ireland: AoIR. Retrieved from http://spir.aoir.org. 
sociology, human-computer interaction, and political economy. In turn it has begun to build a presence within platform studies, game studies, and social media research, given the complex nature of the phenomenon cutting across many traditional definitions of media forms and online media-related behaviours.

The four papers here will each cover an element of understanding live streaming platforms, moving from foundational theory into sociality and consequently into the monetization of these same social practices by both the platform and the live streamers who make a living on it. Specifically, the first talk addresses itself to the sociality of live streaming, which unlike most traditional video media formats enables a rapid live exchange of comments and conversation between live streaming producers and consumers, both enabled by and in turn shaping the affordances of these platforms. The second, third and fourth papers then turn to addressing the monetization of such emerging social behaviours and their entanglements with affective labour on the platform. The second paper presents an overview and first typology of monetization methods in live streaming, focusing in particular on the gamified and "gamblified" elements of making money through the practice, as well as how these practices have evolved through a three-way dialogue between viewers, "streamers", and platforms. The third paper builds on this by examining in detail the on-platform currency of marketleading platform Twitch, known as "Bits", and how the platform captured donations from viewers through the implementation of this currency system. The fourth and final paper will then further develop these critical enquiries into monetization methods and platform dynamics by presenting a number of extremely contemporary developments in this area on Twitch, exploring new the routes for capital flow enabled by new platform infrastructures and technological systems.

In spite of the size and impact of live streaming platforms, the phenomenon remains relatively alien to many scholars when compared to more traditional and studied forms of social media. Drawing on a diverse range of theoretical perspectives including platform studies, science and technology studies, labour studies and discourse analysis, these papers represent numerous directions of enquiry at the forefront of live streaming research.

We now proceed to outline the abstracts for the four constituent talks in this panel (three included in video upload).

\section{THE SOCIAL DEMANDS OF LIVE STREAMING PLATFORM AUDIENCES Nick Bowman, Texas Tech University}

Whether one is live-streaming to an audience approaching zero or an audience approaching tens of thousands (or more; Taylor, 2018), technologies that allow us to broadcast ourselves have brought the social history of gaming to the fore. Videogames can be played in isolation, but the technology has a broad and deep social history (Bowman, et al., 2013; Lin et al., 2018). The earliest gaming technologies were designed with multiple human players in mind (as technological limitations did not allow for simulated intelligent opponents). Games moved quickly into spectated social places, such as bars and taverns-the first PONG game was so stuffed with coins that they jammed the machine's internal mechanisms (Computer History Museum, n.d.). More 
youth-oriented video game arcades developed through the 70 s and $80 \mathrm{~s}$, much to the chagrin of concerned teachers and parents, but fostering a thriving socio-cultural scene around gaming. Advances in network technologies allowed these social structures to carry over to digital spaces, encouraging some to refer to videogames as third places of discourse (Steinkuehler \& Williams, 2006). At the start of the 21st century, most every home gaming console, personal computer, and portable gaming platform allows for both co-located and networked social play.

In this discussion, I examine how gaming-oriented social behaviours have moved onto the Twitch.tv platform, and how the infrastructure of the platform both shapes and is shaped by the fundamentally social dimension of much gaming behaviour. Perhaps harkening back to their early spectated sociality, game streaming on platforms like Twitch has emerged as a deeply social aspect of gaming (Hamari \& Sjoblom, 2017). Although surprising to some who presume the primary entertainment value of video games to be their interactivity, live-streaming video games satisfies one of the core competencies of the earliest gaming developers: the content is "entertain(ing) to the onlooker" (Graetz, 1981). The success of game live streaming platforms demonstrates that there is just not just a market for more carefully-curated gaming videos, such as those on YouTube, but also one where live spectatorship and back-and-forth communication are central. Consalvo et al. (2018) conceptualized tandem play to describe the entertainment derived from audiences watching others play: platforms (such as Twitch) provide and encourage tandem play through direct chat functions through which viewers can help out or suggest things to the streamer. This affordance of the platforms marks them out fundamentally from a one-to-many model of YouTube gaming videos. In a real sense, streaming audiences can be either synchronous or asynchronous (Lin et al, 2018) depending on individual platforms or settings within a particular platform, allowing audience sociality to be variably relevant to the streamer's and the player's own end goals.

From the perspective of interactivity-as-demand (Bowman, 2018), we can unpack how game streamers-those performing for others-are influenced by the experience of using streaming platforms and navigating their infrastructures. Beyond critical discussions of monetization and playbor practices associated with streaming (cf. Woodcock \& Johnson, 2019), the mere presence of audiences facilitated by streaming platforms change how games are experienced, sometimes felt as a form of labor and other times, a performative act of pleasure. For example, Zajonc (1969) argues for a social facilitation effect by which the presence of spectators during performance can dramatically impact said performance. Bowman et al. (2013) tested this, finding that inperson gaming audiences significantly increased a gamer's performance at first-person shooters (when compared to gamers playing in isolation). When replicated using Twitch.tv (synchronous streaming) and YouTube (asynchronous streaming), we were not able to replicate the social facilitation effects-suggesting that mere presence effects might not transfer to digital audiences common to game streaming. Future work might work to more specifically capture platform elements that foster explicit and active spectatorship, with the potential to impact gaming performance in myriad ways.

Notably, even the audience's experience with a game stream can be influenced by the presence of others-a phenomenon known as the intra-audience effect by which the 
affective experience of spectatorship is colored by one's perceived affective response of others (Hocking et al., 1977). In other words, audience pleasure (or displeasure) is boosted when other audience members also express enjoyment (or otherwise). Interactivity appears to be a central component of Twitch's operation as a platform, as live chat provides an avenue for spectators to communicate their impressions of the stream to the streamer and each other, thereby contributing to this intra-audience effect (Lin et al., 2018).

Scholars already engage the social dynamics of game streaming, and this work has greatly informed how we understand social interactions in these places, for example as motivators for engagement (Hilvert-Bruce et al., 2018) as well as barriers to participation (such as the dominance of gendered language in streaming spaces; Ruvalcaba et al., 2018). A (social) demand perspective augments these perspectives extending our focus beyond social interactions and exchanges, and to the psychologically demanding experience of sociality in these spaces. As with the earliest video games, game streaming allows players of various skills to publicly perform their videogame play. Platforms facilitating this play, including Twitch, affect the social arrangements of players and non-players as both reflections of and departures from videogame play's historical sociality.

\section{HOW LIVE STREAMERS ON TWITCH.TV MONETIZE AND GAMIFY THEIR BROADCASTS Mark R Johnson, University of Sydney \& Jamie Woodcock, The Open University}

This paper presents an overview of economic behaviour on dominant market-leading live streaming platform Twitch.tv. Specifically, we are interested in how live streamers profit from their activities - monetization - on the platform, and how they often do so through the implementation of game-like interfaces, competitions, or concepts gamification - which are highly effective in this space. We begin by reviewing relevant literature on both Twitch (Pires \& Simon, 2015; Johnson \& Woodcock, 2017; Ask et al, 2019; etc) and platforms (Gillespie, 2010; Srnicek, 2017; etc), followed by a description of our data and methodology which draws on interview and ethnographic techniques. Over the past four years we have conducted over 100 semi-structured interviews with live streamers of both professional and semiprofessional status. The majority of respondents were in their twenties and from the United States, although nationality was diverse, with most of our respondents hailing from other Global North countries (primarily Canada and within Europe), but also a significant number from the Global South (especially South America), in a ratio of approximately five to one. Around seventy percent of our interviewees were in their 20 s, with almost thirty percent in their $30 \mathrm{~s}$, and only one or two younger or older than those categories. This interview data is coupled with ethnographic findings from several hundred hours of observation at live streaming events attended in person in the US, UK, Germany and Poland, and from 200 live streams viewed each for at least one hour. This ethnographic engagement allows us to see something of the performance of work that streamers perform in their daily activities, which is highly comparable (Woodcock \& Johnson, 2019) to other publicfacing performative jobs (cf. Duffy, 2017) which mobilise affect and emotion, such as fashion (e.g. Wissinger, 2007) or blogging (e.g. Bruns, 2008). By combining interview data and ethnographic work, we are thus able to achieve both a detailed look into the 
lives and actions of particular streamers, and a broader assessment of the culture arising around live streaming and the affordances of streaming platforms, and - in this case - how these shape monetization models.

The core of the paper then addresses seven core monetization methods we identify for live streaming. Subscriptions entail a guarantee to give a monthly amount to a streamer in exchange for the visual distinction of one's username on Twitch. Donations and "cheering" entail either giving the streamer money directly via Paypal, or donating through Twitch, during which the platform takes a cut of the money, but viewers get automatic recognition of their donations. Advertising entails running adverts for corporate products on one's channel. Sponsorships are secured by many live streamers with games companies or other brands, who offer free products or promotion in exchange for highlighting their wares during particular broadcasts. Competitions and targets involve encouraging buy-in from viewers in the hope of winning an individual or global prize. Unpredictable rewards for financial support are the sixth method, drawing on the psychology of gambling and games of unpredictability more broadly to keep people donating in the hope of recognition. Finally, the implementation of monetary "channel games" represent a gamification of the Twitch platform itself, highly appropriate given its primary user market.

We cover each monetization technique in turn, considering what elements of the platform encourage them, how streamers act, how viewers act, and how elements of gaming culture have been skillfully monetized by entrepreneurial live streamers - yet within boundaries and confines laid out by Twitch. In particular, we focus on practices that exist both within and beyond the governance of the platform itself: this analysis of Twitch examines how these economic dynamics influence, and are influenced by, the political, social and cultural relationships of live streaming. The monetization models emerging here have important implications for the entrepreneurial (generally young) individuals trying to build new online careers, as well as for how other platforms (or people on platforms) may choose to monetize. On Twitch itself, meanwhile, the striking profitability of the most successful streamers and Twitch as a whole make it is crucial to interrogate who is winning and losing in financial terms, and why, and how the role of money brushes up against the inherent playfulness of a platform dedicated (primarily) to gaming.

We argue that such a wide variety of monetization methods is possible because the platform is relatively devoid of explicit rules or regulations preventing streamer behaviours, allowing for consistent innovation and change within the broader structure of the live stream. This has led to an ongoing relationship between the platform and its streamers which is both iterative - things are regularly changing and progressing and becoming ever more "optimised" (cf. Partin, 2019) - and recursive - as both parties are influencing the behaviours of the other. The laxity of these restrictions is such that streamers can even run games of chance of debatable legality, although the length of time this situation will continue unabated, or without capture by the platform, remains to be seen. Equally, the norms of Twitch for both its streamers and its viewers contribute significantly to this profusion of monetization methods: viewers are consistently eager to support their favourite streamers and be rewarded (non-financially or financially) in exchange, while aspiring streamers think nothing of encouraging as many donations 
from their viewers as possible. The exchange of money is built so deeply into both the infrastructure and the culture of live streaming that new monetization methods are welcomed by broadcaster and consumer alike. Alongside all of this, the games focus of Twitch also makes it an environment filled with viewers who are highly comfortable with digital play of all different kinds, and thus both able and willing to engage with some of the more gamified monetization methods we have outlined here.

\section{BIT BY (TWITCH) BIT: FROM 'PLATFORM ENVELOPMENT' TO 'PLATFORM CAPTURE' \\ Will Partin, University of North Carolina at Chapel Hill}

As the literature on platforms-defined, in their broadest sense, as digital infrastructures that facilitate interactions between users while also producing and collecting data on these interactions (van Dijck, Poell, \& de Waal 2018)—has grown over the last decade, scholars in a variety of fields have begun to address questions of how platforms evolve as technological objects. Though scholars have long recognized that the technical features of platforms are constantly in flux, the actual processes and trajectories by which platforms evolve over time are comparatively understudied (Helmond, Nieborg, \& van der Vlist, 2019). By problematizing how platforms evolve-that is, by seeing certain modalities of change not as natural or inevitable, but as being shaped by specific contexts-it becomes possible to ask questions about on whose terms and to what effects the technical evolution of platforms occurs.

This paper theorizes "platform capture" as one way that platforms accrue new features though complex interaction with other actors in a platform ecosystem. Platform capture, in my definition, denotes a process in which (1) complementors within a platform marketplace, such as software developers who create applications using boundary resources like APIs, create new features that produce new value for a platform ecosystem by extending its capabilities; (2) platform owners create first-party versions of these features that direct new value to themselves; (3) third-party tools are forced into competition against first-party offerings in a platform marketplace that is biased towards its owners. Platform capture describes a cycle in which the technical architecture of platforms evolves through the exploitation of power asymmetries between platform owners and dependents, raising questions about the monopolistic tendencies of platform ecosystems.

Taken purely as a description of actions by platform owners, platform capture has much in common with what scholars of strategic management call platform envelopment (Eisenmann, Parker, \& Van Alstyne 2010). Yet whereas scholars in this tradition tend to see platform envelopment as a business tactic, this article offers a more critical eye on this practice by reading it in the context of David Nieborg and Thomas Poell's (2018) notion of the platformization of cultural production, a framework that synthesizes insights on platforms not only from business studies but also critical political economy and software studies. This holistic framework systematizes inquiry into platforms to make their impacts on cultural production more visible. By rethinking platform envelopment as platform capture via Poell and Nieborg's framework, it becomes possible to connect this process to broader concerns about the accumulation of power and influence in the information technology sector, as well as technology firms' role in 
producing, maintaining, and exploiting widespread precariousness in contemporary capitalism.

To illustrate platform capture, I consider the history of donation management tools on Twitch, an Amazon-owned platform that hosts user-generated broadcasts of live video game play. Not only is Twitch an important spot for understanding "social media entertainment" (Cunningham and Craig 2019), I argue that it is a useful point for studying how platform architectures gradually acquire new features over time in complex interactions with their dependents.

Taking as a given that platforms "evolve via a complex interplay among users, technologies, infrastructures, organizational structures, and various social, cultural, and economic practices" (Nieborg \& Helmond, 2019: 125), I adopt a holistic view of Twitch that does not reduce its technical evolution to a single cause, but, rather, sees that process as taking place within and through a broader platform ecosystem that does only include Twitch, but also the viewers, streamers, and application developers who engage with the platform in various ways. Likewise, this paper also draws upon textual boundary resources from developers working with Twitch, professional streamers' public statements on donation tools, and Twitch's own announcements. This approach reveals that, like Facebook's own growth into a platform-as-infrastructure, Twitch's own evolution as a technical architecture has been an incremental process, not a revolutionary one, but still evidences Twitch's gradual consolidation of power and influence over its ecosystem. In particular, I demonstrate how the financial precariousness of cultural producers on the platform led to the development of thirdparty donation management services, such as TwitchAlerts, often at significant personal or venture-backed expense (see also Johnson \& Woodcock 2019). These were later supplemented by a first party program, Twitch Bits, which replicated the functionality of third-party donation management tools but was integrated directly into the Twitch user interface, giving the first party-service a significant competitive advantage over thirdparty tools.

While this process could be described as a kind of platform envelopment, the normative stance of scholars in strategic management renders this perspective insensitive to the power dynamics at play in development. In particular, platform envelopment, as theorized, suggests that third-party application developers become competitive with platform owners rather than being interpellated as competitors by the development of new technical features. This orientation towards platform evolution glosses over the power dynamics that are embedded in technical dependency are conditions of possibility for envelopment attacks. Platform capture, by contrast, draws attention to how the power asymmetries between application developers and Twitch were exploited by Twitch in service of the new development of features, raising questions about the concentration of power and capital in large technology corporations and the potentially deleterious impacts on innovation, an observation that has implications far beyond Twitch.

\section{CAPITAL FLOW AND TEMPORAL POLITICS ON TWITCH: AN EXAMINATION OF NEW FEATURES AND AFFORDANCES Nathan Jackson, University of New South Wales}


Since mid-2018, the livestreaming platform Twitch has seen the advent of a number of new features influencing how users spend their time and money. In this paper, I will introduce four of these features and examine their associated social and technological affordances. Namely, I will examine how they guide users to spend their money in particular ways and at particular times - that is, how they moderate capital flow - and how they demonstrate a shifting balance between the value of viewers' time and capital in favour of the former. Ultimately, I will interrogate how these features interact to add layers of complexity within and around the already complex economic structure of the platform. In this paper, I will update the taxonomy of avenues for Twitch monetisation and associated gamification practices produced by Johnson and Woodcock (2019). I will also extend discussions of streamer practices and associated labour (Johnson et al., 2019; Woodcock \& Johnson, 2019) as streamers decide how to integrate these features into their performances without adversely affecting their relationship with their viewers (Walker, 2014; Taylor, 2018; Sjöblom et al, 2019). As these features affect the flow of time and capital spent on the platform, their close analysis will enable assessment of the rapidly-evolving relationships between the social behaviour, technical infrastructures, and economic imperatives of the platform.

Specifically, I will discuss the emergence of 'subscription gifting', 'Channel Points', 'emote gifts', and 'Hype Trains', each of which represents a new constellation of socialtechnical economic elements on the platform. In the first case, when a Twitch user subscribes to a streamer, they demonstrate support for the streamer through a tiered monthly fee split between the streamer and Amazon. A subscriber receives a badge for display next to their username in that streamer's chat, as well as a suite of subscriberspecific emoticons, called emotes, for use across the platform. As suggested by the name, the oldest feature on this list - released late 2018 - subscription gifting is the ability for one Twitch user to pay for a (single month) channel subscription for another. Subscription gifting has both economic and social impacts, for instance acting as a gesture of welcome for newcomers. If the recipient renews their subscription, this donation becomes a source of regular income for the streamer. In this way, gift subscriptions creatively exemplify the relationship between the market-driven and gift economy elements of the digital economy (Terranova, 2000).

A second and newer feature, trialled in late 2019 and implemented early 2020, Channel Points is an opt-out feature that rewards time spent in a stream. The feature formalises pre-existing gamification practices implemented by some streamers to incentivise viewers to spend time in their streams by accumulating channel-specific currency (Siutila, 2018). Viewers accrue points primarily by watching, actively watching (regularly clicking a button below chat), and viewing consecutive streams. Channel Points can be redeemed for rewards that streamers can customise, thereby enacting creativity to encourage sustained viewing. The streamer can commit to perform specific acts, change the stream title or music playlist for points. Whilst clearly emphasising the value of viewing time, the ties to capital emerge when examining the default rewards, which include the ability to unlock or modify a chosen subscriber emote for one day. This heavily complicates the existing economics of the platform and has been met with mixed reactions. Some streamers openly criticise the feature, claiming it removes the need for viewers to subscribe in order to access emotes, which reduces the value of the 
emotes. Others appreciate that this enables viewers without the means/ability to subscribe to engage more deeply as a member of the stream collective, reflecting the time that they commit to the channel. As such, Channel Points illuminate how heavily contested the economic structure of the platform is among users.

The third feature, emote gifts, is event-based and has occurred increasingly since Pride Month in May 2019. During event periods, including the most recent Pride Month, Christmas Holiday period, and Valentine's Day, bit donations (Twitch currency) and (gift) subscriptions permanently unlock emotes from an event-themed collection for the donor, as well as gifting one of those emotes to a number of other viewers. These events impact capital flow and user engagement in three ways. Firstly, they act as an incentive for viewers to spend money on the platform. This was furthered in the case of Pride Month, when a percentage of bit donations was committed to LGBTQ youth crisis support through The Trevor Project. Secondly, these events encourage viewers to spend more time on the platform - and in particular with more generous communities in the hopes of earning more emotes as gifts. Lastly, they encourage users to spend more money during particular times of year.

This temporal influence over spending patterns extends into the final and newest feature I will present: Hype Trains. Drawn from the streamer-driven practice of the same name, viewers are challenged to chain donations and/or subscriptions before a timer runs out. The Hype Train activates when a customisable number of distinct donation or subscription events occur within a particular length of time. Viewers continue to 'build hype' by donating and subscribing until a bar above chat fills - at which point the Hype Train levels up. This continues until either Level 5 or the timer runs out, at which point every participant receives a random level-dependent emote. Again, this feature has been met with mixed responses, with some stream communities relabelling it as the 'Scam Train', solidifying the ambivalence among users towards the platform's monetisation strategies.

I draw upon data from an ongoing ethnography of Twitch designed to examine the construction and performance of streaming personas. I will bring these features into conversation to emphasise their effect on user practice, the value of emotes, and the feedback loop between social practices and technological features. This will culminate in a more nuanced understanding of capital flow and the value of time on the platform, as well as users' ambivalence towards Twitch's constant innovation in monetisation practices.

\section{References}

Akrich, M. (1992). The de-scription of technological objects. In W. E. Bijker \& J. Law (Eds.), Shaping technology/building society: Studies in sociotechnical change. Cambridge, MA: MIT Press.

Ask, K., Spilker, H. S., \& Hansen, M. (2019). The politics of user-platform relationships: Co-scripting live-streaming on Twitch. tv. First Monday, 24(7). 
Bowman, N. D. (2018). The demanding nature of video game play. In N. D. Bowman (Ed.), Video games: A medium that demands our attention (pp. 1-24). New York: Routledge.

Bowman, N. D., Weber, R., Tamborini, R., \& Sherry, J. L. (2013). Facilitating game play: How others affect performance at and enjoyment of video games. Media Psychology, 16(1), 39-64.

Bruns, A. (2009). The user-led disruption: Self-(re)broadcasting at Justin.tv and elsewhere. In EurolTV'09: Proceedings of the seventh European Interactive Television Conference, Leuven, Belgium, June 3-5, 2009.

Bruns, Axel . 2008. Blogs, Wikipedia, Second Life, and beyond: From Production to Produsage. New York: Peter Lang.

Burroughs, B. E. (2015). Streaming media: Audience and industry shifts in a networked society (Doctoral dissertation, University of lowa). Retrieved from http://ir.uiowa.edu/etd/1833.

Computer History Museum (n.d.). PONG. Retrieved from https://www.computerhistory.org/revolution/computer-games/16/183.

Consalvo, M., Begy, J., Ganzon, S. C., \& Scully-Blaker, R. (2018). Tandem play: Theorizing sociality in single-player gameplay. In N. D. Bowman (Ed.), Videogames: A medium that demands our attention.

Duffy, B. E. (2017). (Not) getting paid to do what you love: Gender, social media, and aspirational work. New Haven, CT: Yale University Press.

Egli, E., \& Meyers, L. S. (1984). The role of videogame playing in adolescent life: Is there reason to be concerned? Bulletin of the Psychonomic Society, 22(4), 309-312.

Eisenmann, T., Parker, G., \& Alstyne, M. V. (2010). Platform Envelopment (Working Paper). Harvard Business School, 07.

Gillespie, T. (2010). The Politics of "Platforms". New Media \& Society, 12 (3): 347-364.

Graetz, J. M. (1981, August). The origin of Spacewar! Creative Computing Magazine.

Hamari, J., \& Sjöblom, M. (2017). What is esports and why do people watch it? Internet Research, 27.

Helmond, A., Nieborg, D. B., \& van der Vlist, F. N. (2019). Facebook's evolution: Development of a platform-as-infrastructure. Internet Histories, 3, 123-146.

Hilvert-Bruce, Z., Neill, J. T., Sjöblom, M., \& Hamari, J. (2018). Social motivations of live-streaming viewer engagement on Twitch. Computers in Human Behavior, 84, 5867. 
Hocking, J. E., Margreiter, D. G., \& Hylton, C. (1977). Intra-audience effects: A field test. Human Communication Research, 3(3), 243-249.

Johnson, M. R., \& Woodcock, J. (2017). 'It's like the gold rush': the lives and careers of professional video game streamers on Twitch. tv. Information, Communication \& Society, 22(3), 336-351.

Johnson, M.R., Carrigan, M, and Brock, T. (2019). The imperative to be seen: The moral economy of celebrity video game streamers on Twitch.tv. First Monday, 24(8).

Johnson, M.R., Woodcock, J. (2019). "And Today's Top Donator is": How Live Streamers on Twitch.tv Monetize and Gamify Their Broadcasts. Social Media + Society, 5(4): 1-11.

Lin, J-S., Bowman, N. D., Lin, S-F., Chen. S. (2019). Setting the digital stage: Defining game streaming as an entertainment experience. Entertainment Computing, 31.

Nieborg, D. B., \& Helmond, A. (2019). The political economy of Facebook's platformization in the mobile ecosystem: Facebook Messenger as a platform instance. Media, Culture \& Society, 41, 196-218.

Nieborg, D. B., \& Poell, T. (2018). The platformization of cultural production: Theorizing the contingent cultural commodity. New Media \& Society, 20, 4275-4292.

Pires, K., \& Simon, G. (2015). YouTube live and Twitch: A tour of user-generated live streaming systems. 6th ACM multimedia systems conference, Portland, Oregon, 225230.

Ruvalcaba, O., Schulze. J., Kim. A., Berzenski, S. R., \& Otten, M. P. (2018). Women's experiences in esports: Gendered differences in peer and spectator feedback during competitive video game play. Journal of Sport and Social Issues.

Siutila, M. (2018). The gamification of gaming streams. GamiFIN Conference 2018, Levi, Finland, 131-140.

Sjöblom, M., Törhönen, M., Hamari, J., and Macey, J. (2019). The ingredients of Twitch streaming: Affordances of game streams. Computers in Human Behavior, 92: 20-28.

Srnicek, N. (2017). Platform Capitalism. Cambridge: Polity.

Steinkuehler, C. A., \& Williams, D. (2006). Where everybody knows your (screen) name: Online games as "third places." Journal of Computer-Mediated Communication, 11(4).

Taylor, T. L. (2018). Watch me play: Twitch and the rise of game live-streaming. Princeton: NJ: Princeton University Press. 
Terranova, T. (2000). Free Labor: Producing Culture for the Digital Economy. Social Text 63, 18(2): 33-58.

Van Dijk, J, T Poell and M De Waal (2019): The Platform Society: Public Values in A Connective World. Oxford: Oxford University Press.

Walker, A. (2014). Watching Us Play: Postures and Platforms of Live Streaming. Surveillance \& Society, 12(3): 437-442.

Wissinger, Elizabeth . 2007. "Modelling a Way of Life: Immaterial and Affective Labour in the Fashion Modelling Industry." Ephemera 7 (1): 250-69.

Woodcock, J and Johnson, M.R. (2019). The Affective Labor and Performance of Live Streaming on Twitch.tv. Television \& New Media, 20(8): 813-823.

Zajonc, R. B. (1965). Social facilitation. Science, 149 (269-275). 